\title{
Rubeosis capsulare: um caso incomum de rubeosis iridis em paciente com implante de lente intra-ocular - Relato de caso
}

\author{
Rubeosiscapsulare: An unusual caseof rubeosis iridis in a patient with posterior intra- \\ ocular lens implantation - Case report
}

\author{
Alexandre A. Marques Rosa ${ }^{1}$ \\ Eduardo Cunha de Souza ${ }^{2}$ \\ Breno Barth ${ }^{3}$ \\ Walton Nosé ${ }^{4}$
}

${ }^{1}$ Médico Estagiário do Setor de Retina e Vítreo do Departamento de Oftalmologia da Faculdade de Medicina da Universidade de São Paulo.

${ }^{2}$ Médico Assistente do Setor de Retina e Vítreo do Departamento de Oftalmologia da Faculdade de Medicina da Universidade de São Paulo.

${ }^{3}$ Médico Pós-Graduando do Departamento de Oftalmologia da Faculdade de Medicina da Universidade de São Paulo.

${ }^{4}$ Professor Livre Docente de Oftalmologia da Escola Paulista de Medicina - UNIFESP.

Endereço para correspondência: Alexandre A. M. Rosa - Rua Afonso de Freitas 66/Ap. 51 - São Paulo (SP) CEP 04006-050

E-mail: alexandre ros@hotmail.com

Recebido para publicação em 02.10.2001

Aceito para publicação em 04.03.2002

\section{RESUMO}

Os autores relatam o caso raro de paciente diabético com rubeosis iridis submetido à cirurgia de catarata com implante de lente intra-ocular, em que houve o crescimento de neovasos na cápsula posterior do cristalino.

Descritores: Neovascularização, patológica/etiologia; Diabetes mellitus/complicações; Lentes intra-oculares; Complicações pós-operatórias/etiologia; Extração de catarata; Doenças da íris/etiologia

\section{INTRODUÇ̃̃̃O}

A extração da catarata com implante de lente intra-ocular, através da técnica de facoemulsificação ou extracapsular, é um dos procedimentos cirúrgicos mais realizados em oftalmologia.

Com as novas modalidades de tratamento e recursos disponíveis obteve-se um aumento na sobrevida dos indivíduos com diabetes melitus ${ }^{(1)}$. Pacientes diabéticos têm uma maior prevalência de opacidades cristalinianas $^{(2)}$, e desenvolvem catarata em idade mais precoce que os não diabé$\operatorname{ticos}^{(3)}$. A catarata nestes doentes, além de causar redução da acuidade visual, dificulta o exame de fundo de olho e a fotocoagulação retiniana.

Estudos demonstraram que pacientes diabéticos estão sujeitos a um número maior de complicações que os não diabéticos, principalmente inflamação pós-operatória mais acentuada ${ }^{(4)} \mathrm{e}$, conseqüente, pior acuidade visual ${ }^{(5)}$.

Os autores relatam o caso de um paciente com neovascularização iriana e implante de lente intra-ocular, com a presença de neovasos na cápsula posterior do cristalino, condição denominada de rubeosis capsulare.

\section{RELATO DE CASO}

AM, 82 anos, masculino, branco, veio para exame oftalmológico, no Ambulatório de Oftalmologia do Hospital das Clínicas da Faculdade de Medicina da Universidade de São Paulo, com queixa de enxergar "galhos de árvore" na frente do olho esquerdo há 3 meses.

Em relação aos antecedentes mórbidos pessoais, referia diabetes melitus tipo II há 15 anos de controle irregular. Quanto aos antecedentes oculares, havia sido submetido à cirurgia de extração extracapsular da catarata com implante de lente intra-ocular, em outro serviço, há 7 meses.

Ao exame oftalmológico, a acuidade visual com a melhor correção era de 0,4 e conta dedos a 3 metros nos olhos direito e esquerdo, respectivamente. 
A pressão intra-ocular era de $16 \mathrm{mmHg}$ no olho direito e $30 \mathrm{mmHg}$ no olho esquerdo. A motilidade ocular extrínseca e os reflexos pupilares não apresentavam anormalidades.

Na oftalmoscopia do olho direito, observávamos uma retinopatia diabética não proliferativa moderada com atrofia do epitélio pigmentário retiniano na região macular. No olho esquerdo, havia uma retinopatia diabética proliferativa com características de alto risco.

A biomicroscopia do olho direito não apresentava alterações, porém no olho esquerdo havia uma lente intra-ocular centrada com opacificação da cápsula posterior e rubeosis iridis, onde se podia notar um vaso sangüíneo crescendo a partir do estroma iriano, atravessando o furo para posicionamento da lente e crescendo sobre a cápsula posterior, atrás da lente intra-ocular (Figuras 1, 2 e 3).

\section{DISCUSSÃO}

Uma piora da retinopatia diabética acontece em grande parte dos casos após facectomia ${ }^{(1,6)}$.

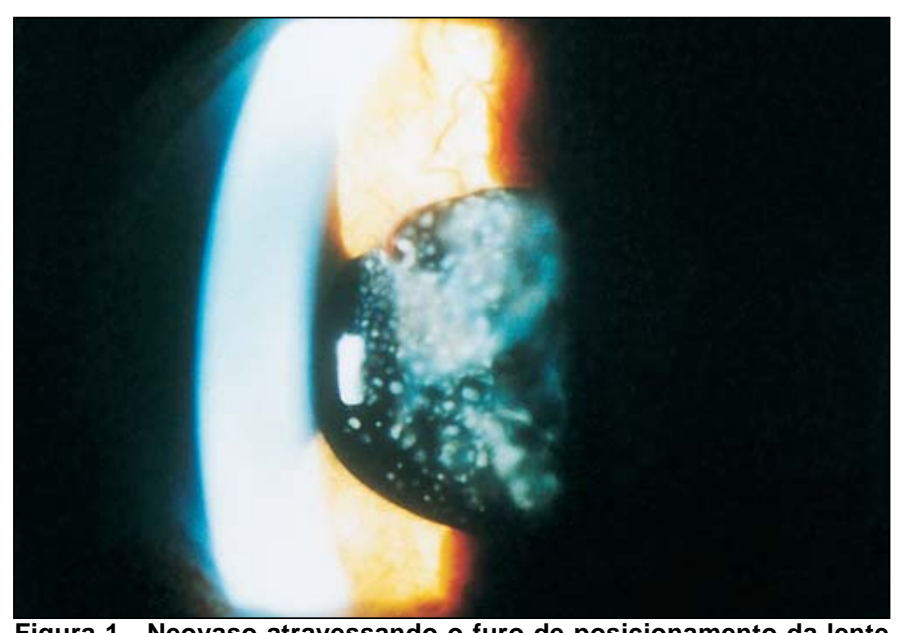

Figura 1 - Neovaso atravessando o furo de posicionamento da lente intra-ocular

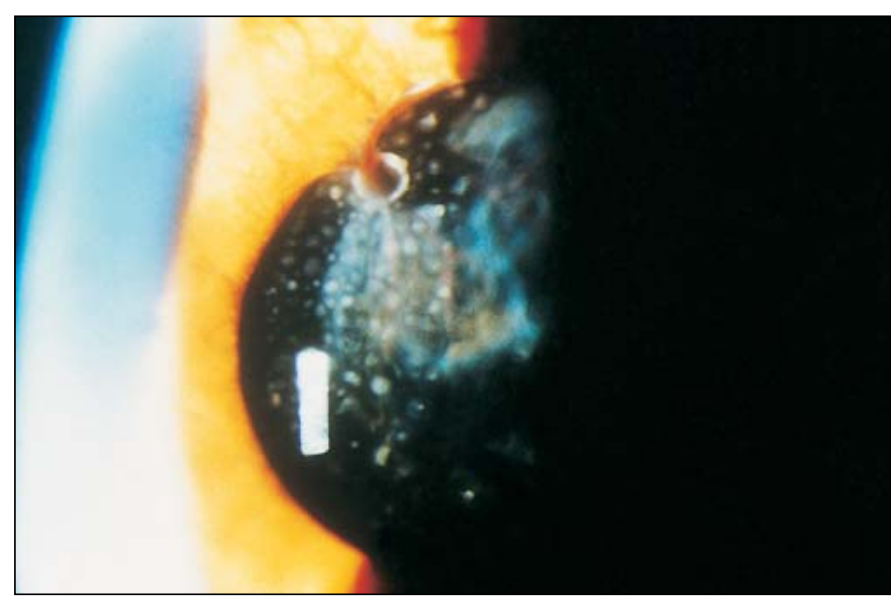

Figura 2 - Maior aumento. Neovaso atravessando o furo de posicionamento da lente intra-ocular

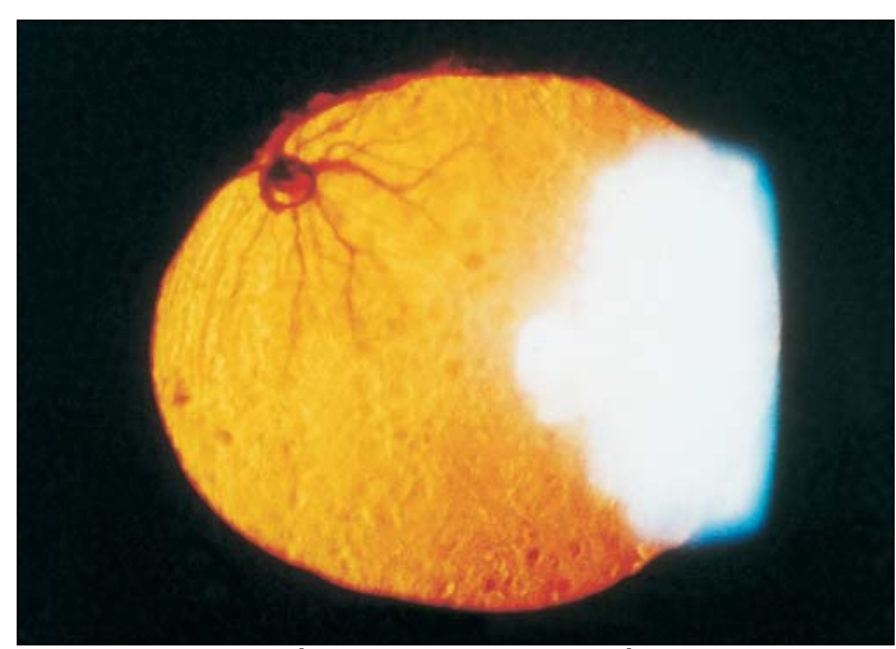

Figura 3 - Neovaso na cápsula posterior visto através do reflexo vermelho

Muitos autores têm tentado identificar os fatores de risco para o prognóstico visual e progressão das alterações retinianas após cirurgia de catarata em pacientes com diabetes melitus. Olhos diabéticos têm mais alterações de segmento anterior, como cristalinos maiores ${ }^{(7)}$, curvatura anterior do cristalino mais acentuada e uma câmara anterior mais rasa ${ }^{(8)}$, principalmente aqueles com retinopatia. Além disso, são mais suscetíveis ao trauma cirúrgico, apresentando miose per-opertária mais pronunciada ${ }^{(9)}$, maior intensidade de "flare" pósoperatório na câmara anterior ${ }^{(10)}$, elevação transitória da pressão intra-ocular ${ }^{(11)}$ e uma maior incidência de edema cistóide de mácula angiográfico ${ }^{(12)}$.

O glaucoma neovascular é uma das complicações finais da retinopatia diabética proliferativa, sua incidência vem diminuindo progressivamente nos últimos anos devido ao melhor controle dos pacientes diabéticos e o tratamento sistemático das retinopatias proliferativas através da panfotocoagulação retiniana com laser de argônio ${ }^{(13)}$.

A neovascularização iriana tem sido chamada de rubeosis iridis desde 1928, e desenvolve-se rapidamente no período pós-operatório a despeito de um bom controle metabólico do diabetes e de um quadro retiniano estável ${ }^{(14)}$. Em geral, a perda visual nestes pacientes costuma ser grave ${ }^{(14)}$.

A rubeosis iridis inicia-se separadamente na zona pupilar, onde existem muitos capilares a partir dos quais os neovasos podem originar-se. Posteriormente, estende-se por todo o estroma iriano.

Eifrig e colaboradores relataram 2 casos de rubeosis iridis, em pacientes diabéticos, com neovascularização dentro da cápsula posterior do cristalino, uma condição que denominaram de rubeosis capsulare ${ }^{(15)}$.

Acreditamos que os neovasos necessitam de um substrato orgânico para crescer, motivo pelo qual não crescem sobre a superfície da lente intra-ocular, ou talvez a presença de mediadores vasoproliferativos (como o VEGF) no corpo vítreo anterior agissem como fator quimiotático. Contudo, estas hipóteses necessitam de confirmação através de estudos histopatológicos. 


\section{ABSTRACT}

The authors report a rare case of a diabetic patient with rubeosis iridis who had cataract extraction with intraocular lens implantation, in whom neovascularization developed within the posterior lens capsule.

Keywords: Neovascularization, pathologic/etiology; Diabetes mellitus/complications; Lenses, intraocular; Postoperative complications/etiology; Cataract extraction; Iris diseases/etiology

\section{REFERÊNCIAS}

1. Stanila A. Diabetes and extracapsular extraction of the crystalline lens. Oftalmologia 1996;40:377-82.

2. Klein BE, Klein R, Moss SE. Prevalence of cataracts in a population-based study of persons with diabetes mellitus. Ophthalmology 1985;92:1191-6.

3. Nielsen NV, Vinding T. The prevalence of cataract in insulin-dependent and non-insulin dependent diabetes mellitus. Acta Ophthalmol (Copenh) 1984;62:595-602.

4. Cunnliffe IA, Flanagan DW, George ND, Aggarwaal RJ, Moore AT. Extracapsular cataract surgery with lens implantation in diabetics with and without proliferative retinopathy. Br J Ophthalmol 1991;75:9-12.
5. Benson WE, Brown GC, Tasman W, McNamara JA, Vander JF. Extracapsular cataract extraction with placement of a posterior chamber lens in patients with diabetic retinopathy. Ophthalmology 1993;100:730-8.

6. Vignanelli M. Progression of diabetic retinopathy following cataract extaction. Klin Monatsbl Augenheilkd 1990;196:334-7.

7. Brown N, Hungerford J. The influence of the size of the lens in ocular disease. Trans Ophthalmol Soc UK 1982;102:359-63.

8. Sparrow JM, Bron AJ, Phelps Brown NA, Neil HA. Biometry of the crystalline lens in late onset diabetes: the importance of diabetic type. Br J Ophthalmol 1992;76:428-33.

9. Zaczek A, Zetterström C. Cataract surgery and pupil size in patients with diabetes mellitus. Acta Ophthalmol Scand 1997;75:429-32.

10. Zaczek A, Zetterström C. Aqueous flare intensity after phacoemulsification in patients with diabetes mellitus. J Cataract Refract Surg 1998;24:1099-104.

11. Kodama T, Hayasaka S, Setogawa T. Plasma glucose levels, postoperative complications, and progression of retinopathy in diabetic patients undergoing intra-ocular lens implantation. Graefes Arch Clin Exp Ophthalmol 1993; 231:439-43.

12. Menchini U, Bandello F, Brancato R, Camesasca FI, Galdini M. Cystoid macular oedema after extracapsular cataract extraction and intraocular lens implantation in diabetic patients without retinopathy. $\mathrm{Br} \mathrm{J}$ Ophthalmol 1993;77:208-11.

13. Detry-Morel M. Neovascular glaucoma in the diabetic patient. Bull Soc Belge Ophtalmol 1995;256:133-41.

14. Sadiq SA, Chatterjee A, Vernon AS. Progression of diabetic retinopathy and rubeotic glaucoma following cataract surgery. Eye 1995;9:728-38.

15. Eifrig DE, Hermsen V, McManus P, Cunningham R. Rubeosis capsulare. J Cataract Refract Surg 1990;16:633-6.

\title{
XXVIII Congresso da Sociedade Brasileira de Retina e Vítreo
}

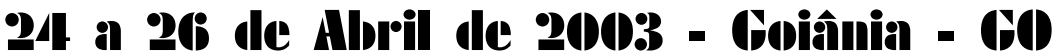

\section{Promoção:}

Sociedade Brasileira de Retina e Vítreo

INFORMAÇÕES: Tel/Fax.: (62) 285-5272

Tel: (62) 285-5827 (Sra. Jalma/Jany)

E-mail: retina2003@cbco.com.br 